\title{
Positive and Negative Online Experiences and Loneliness in Peruvian Adolescents During the COVID-19 Lockdown*
}

\author{
Lucía Magis-Weinberg $^{1 *}$, Christopher L. Gys ${ }^{1}$, Estelle L. Berger ${ }^{2}$, Sarah E. Domoff ${ }^{3}$, Ronald E. \\ Dahl $^{4}$ \\ ${ }^{1}$ Institute of Human Development, University of California, Berkeley \\ ${ }^{2}$ Department of Psychology, University of Oregon \\ ${ }^{3}$ Department of Psychology, Central Michigan University \\ ${ }^{4}$ School of Public Health, University of California, Berkeley
}

\begin{abstract}
Author Note
Corresponding author: Lucía Magis-Weinberg, M.D., Ph.D., Institute of Human Development, University of California, Berkeley, 2121 Berkeley Way, 94720-1690, Berkeley, CA, (415) 42582 46, email: l.magisweinberg@,berkeley.edu

Acknowledgements: The authors appreciate Karina Valdespino's assistance with literature review. The authors would like to thank adolescents and their families for their involvement in the study as well as our school partners who made data collection possible. This research was supported by funding from Carlos Rodriguez-Pastor and the Bezos Family Foundation.

* Currently in press in the Journal of Research on Adolescence.
\end{abstract}




\title{
ADOLESCENT LONELINESS AND ONLINE SOCIAL EXPERIENCES IN COVID-19
}

\begin{abstract}
Global COVID-19 lockdowns have disrupted adolescents' in-person social networks, increasing likelihood of loneliness. Social media can help adolescents maintain and develop peer relationships across distance. In this short longitudinal study with 735 Peruvian adolescents (ages:11-17) from low-to-middle income urban settings, we investigated whether online experiences relate to loneliness during initial stages of lockdown. Loneliness remained constant between week six and eleven of lockdown, was higher for females and similar across schoolgrades. Positive and negative online experiences were more frequent for older students, and females experienced more negative online experiences than males. Greater positive online experiences related to lower loneliness, with the reverse pattern for negative online experiences. Our results suggest that positive online experiences may mitigate loneliness during physical isolation.
\end{abstract}

Keywords: social media, adolescents, loneliness, COVID-19, Global South 
ADOLESCENT LONELINESS AND ONLINE SOCIAL EXPERIENCES IN COVID-19

\section{Positive and Negative Online Experiences and Loneliness in Peruvian Adolescents During the COVID-19 Lockdown}

As part of Perú's national response to the COVID-19 pandemic, families were bound to their homes in the early weeks of lockdown, with the exception of authorization for one adult in the household to leave to buy essential items and special permissions for essential workers. While peers and friends are usually an important source of in-person support for adolescents (Orben et al., 2020), this was completely replaced by parents and siblings at home. Adolescents, who were already increasingly communicating with peers over social media to complement offline relationships before the pandemic (Lenhart, 2015), are currently fully dependent on these platforms to interact with peers and friends (Parents Together, 2020). In this study, we focus on how perceived online positive (i.e., social support through social media) and negative experiences (i.e., social negativity in social media interactions) relate to perceptions of loneliness for adolescents in Perú over five weeks of COVID-19 lockdown.

\section{Adolescence, a Sensitive Developmental Period for Social Connectedness, Might Be a Period of Vulnerability to Loneliness during Lockdown}

Adolescence is a developmental period marked by relational changes and restructuring of social networks (Helsen et al., 2000), with social reorientation away from parental influence towards peers and friends (B. Bradford Brown, 1990, 2004; Nelson et al., 2015). Characterized by heightened risk for onset of affective disorders, adolescence is also a time when peer relations play a role in the onset and maintenance of psychopathology (Choukas-Bradley \& Prinstein, 2014). Social connectedness during adolescence is beneficial for socioemotional functioning across diverse outcomes (e.g., mental health, academic performance; Orben et al., 2020). Social connectedness is also key for engaging in social learning experiences and social value learning during this key time of identity development (Dahl et al., 2018).

The lockdown measures following the outbreak of COVID-19 in Spring 2020 have presented new barriers to social connectedness, putting adolescents at risk of social isolation and loneliness (Loades et al., 2020). During quarantine, social media platforms have taken center stage as a lifeline for maintaining relationships across distance (Ellis et al., 2020). Past research suggests that social media may be able to ameliorate the toll of physical isolation on well-being by facilitating feelings of social support, social capital, and belonging in adolescents (Allen et al., 2014; Lin et al., 2020; Orben, 2020). With continued COVID-19-related social distancing 


\section{ADOLESCENT LONELINESS AND ONLINE SOCIAL EXPERIENCES IN COVID-19}

mandates, as well as a growing technological infrastructure supporting remote learning and socializing (e.g., Zoom, Instagram), it is imperative to disentangle the ways in which digital technology use may be protective or put adolescents at risk for developing mental health problems associated with physical distancing and social isolation (Loades et al., 2020; Orben et al., 2020).

\section{Loneliness}

While social isolation is the objective measure of an individual's social contact, it is not necessarily the same as loneliness, which is a subjective experience due to a perceived mismatch between desired and actual social interactions (Perlman \& Peplau, 1981; Wang et al., 2017). Thus, although lockdown has not necessarily severed peer relationships and may have, in fact, increased adolescents' time with family, loneliness may still arise from drastic shifts in social dynamics (Laursen \& Hartl, 2013) occurring during in an abrupt transition to remote education. The pursuit of autonomy and independence from parents, combined with the salience of peer relations during adolescence, may place adolescents at risk of accentuated loneliness due to the absence of in-person connection with peers (Heinrich \& Gullone, 2006).

In addition, gender-related differences in prevalence of loneliness during adolescence are an important consideration. While one recent meta-analysis found no significant association between gender and loneliness during adolescence (Maes et al., 2019), other studies have yielded conflicting findings depending on how loneliness was measured. Specifically, when loneliness is asked about more directly (e.g., using the word "lonely"), adolescent females tend to report higher loneliness than males (von Soest et al., 2020; Heinrich \& Gullone, 2006); however, when loneliness is measured more indirectly (e.g., social loneliness), such as with the UCLA Loneliness Scale (Russell, 1982; Russell, 1996), these gender differences do not emerge or males

report higher loneliness than females (Heinrich \& Gullone, 2006; Barreto et al., 2020). In light of these findings, consideration of gender in the investigation of loneliness during adolescence is especially important.

While it has been observed that older people report loneliness less frequently than younger people (Barreto et al., 2020), few studies have explored age differences within adolescence. The rapid biological changes during puberty and the many social transitions occurring around this time (e.g., schools, friend groups, identity) may confer vulnerability to adolescents (Qualter et al., 2012, 2015). Indeed, some empirical studies support this notion. 


\section{ADOLESCENT LONELINESS AND ONLINE SOCIAL EXPERIENCES IN COVID-19}

While age did not emerge as a significant predictor of loneliness in one study sampling adolescents from age 14 to 18 (Ellis et al., 2020), another study observed a rise in loneliness from early to late adolescence, peaking at age 19 (Shovestul et al., 2020). Another study of Norwegian adolescents and young adults found that emotional (as opposed to social) loneliness rose from early adolescence into mid-20s (von Soest et al., 2020). Thus, controlling for possible age-related differences when analyzing the impact of online interactions on loneliness will be important in future research.

\section{Social Support and Loneliness}

Perceived social support is linked to psychosocial well-being in developing populations (Chu et al., 2010), especially during adolescence due to the heightened sensitivity to social contexts (Blakemore \& Mills, 2014; Klineberg et al., 2006). Subjective or perceived social support refers to the perception of the emotional, instrumental, informational and appraisal benefits a social network may confer (Demaray \& Malecki, 2002), while objective social support may refer to the size and complexity of one's social network and the frequency and nature of contact with those in this network (George et al., 1989). As independent sources of perceived social support, relationships with peers and parents exhibit different trajectories during adolescence, with perceived peer support increasing and perceived parental support decreasing with age (Cauce et al., 1990; Furman \& Buhrmester, 1992; Helsen et al., 2000; Meeus, 1989). Thus, in the face of COVID-19-related physical distancing and social isolation, perceived social support could help counter feelings of loneliness during the pandemic, but, in part, could depend on the source of perceived support in question (e.g., parents vs. peers).

\section{Social Support Online}

In a situation of extreme social isolation from peers, indirect strategies for social connection are important sources of support and well-being (Gardner et al., 2005), amplifying the role that social media already has in nurturing relationships with peers and friends (Uhls et al., 2017; Valkenburg \& Peter, 2008; Vannucci \& McCauley Ohannessian, 2019). Recent surveys in the USA before the pandemic did not show, however, that adolescents prioritized using social media to feel less alone or get support, relative to reinforcing relationships with offline friends (Rideout \& Fox, 2018). However, due to the current COVID-19 restrictions on inperson support, it is possible that social media has assumed a more central role for getting social support and also for developing new relationships. 


\section{ADOLESCENT LONELINESS AND ONLINE SOCIAL EXPERIENCES IN COVID-19}

Online social interactions have implications for psychological functioning, particularly for adolescents during the pandemic who have been cut-off from connecting with peers and friends in-person. While the research on social media and emotional outcomes has yielded inconsistent or marginally negative associations, the link with social outcomes (e.g., social connectedness, social capital) has been positive in previous studies (Orben, 2020; Allen, et al., 2014). Online contexts might provide social positivity, including social support, but at the same time expose users to social negativity (Kent de Grey et al., 2019). The interpersonal-connectionbehaviors framework (Clark et al., 2018) posits that social media is beneficial when it allows for meaningful social connection, and harmful to the extent that these interactions might promote social comparisons or create feelings of social isolation. In line with this framework, the association between social media and loneliness is inconsistent (Dienlin et al., 2017; OECD, 2019; Primack et al., 2017; Primack et al., 2019). Inconsistencies relate to how individuals use social media, which likely influences the impact that social media use may have on well-being (see Kross et al., 2020 and Odgers \& Jensen, 2020 for reviews).

\section{Positive Online Experiences}

Positive online experiences - or interactions promoting support in the form of information, belonging, social capital, or emotional wellbeing - have been shown to promote social connectedness (Clark et al., 2018; Kent de Grey et al., 2019). Social media may be a vehicle for the perceived social support, social capital, and social belonging that would normally be provided by in-person interaction (Best et al., 2014; Davis, 2012; Odgers \& Jensen, 2020; Yau \& Reich, 2017). A recent narrative review highlights that the link between social media and social outcomes (e.g., social connectedness, social capital) has been positive in previous studies (Orben, 2020), especially when social media use entails active communication with friends and family (Burke \& Kraut, 2016). Positive online experiences may alleviate loneliness in particular. Online tools have allowed lonely individuals to make friends online and to be more satisfied with their friendships (Morahan-Martin \& Schumacher, 2003). In a majority Dutch adolescent sample, instant messenger chatting predicted less loneliness six months later (van den Eijnden et al., 2008). Another study of Belgian adolescents found that using Facebook to make new friends reduced peer-related loneliness, while Facebook use related to compensating for weak social skills increased loneliness (Teppers et al., 2014). In contrast, in a sample representative of the German population ages 16 and older, increases in online communication were not related to a 


\section{ADOLESCENT LONELINESS AND ONLINE SOCIAL EXPERIENCES IN COVID-19}

change in loneliness, but were related to life-satisfaction (Dienlin et al., 2017). These inconsistencies highlight how the quality of online experiences may relate to the change in loneliness over time, especially among understudied adolescent populations in the Global South.Another study of Belgian adolescents found that using Facebook to make new friends reduced peer-related loneliness, while Facebook use related to compensating for weak social skills increased loneliness (Teppers et al., 2014). In contrast, in a sample representative of the German population ages 16 and older, increases in online communication were not related to a change in loneliness, but were related to life-satisfaction (Dienlin et al., 2017). These inconsistencies highlight the need for further investigation of how the quality of online experiences may moderate the change in loneliness over time, especially among understudied adolescent populations in the Global South.

\section{Negative Online Experiences}

Negative online social experiences — or interactions that engender such feelings as exclusion or rejection (Kent de Grey et al., 2019) — have been associated with negative long-term impacts on mental health including stress, depression, and anxiety (Davila et al., 2012; Hampton et al., 2016; Primack et al., 2017). In a sample of US adolescents, cybervictimization predicted depression three years later (Rose \& Tynes, 2015). Before the pandemic, an association between social media use and loneliness was documented in adults (Clark et al., 2018). This link is likely bidirectional: increased Facebook use has been associated with more loneliness (Song et al., 2014), but also, among US college students, lonely participants reported using more online communications and turning online for social support (Morahan-Martin \& Schumacher, 2003). Social media platforms also facilitate "social snacking" where non-interactive social experiences might give the illusion of satisfying social needs (Gardner et al., 2005), for example, by passively viewing profiles (Carpenter et al., 2011). Passive, superficial connections on social media can displace meaningful interpersonal connection when opportunities to connect in person exist (Green et al., 2005). However, in the present circumstances, social media present the main avenue for not only superficial, but also more meaningful interactions with others.

Compounding the mixed finding on associations between social media and well-being and loneliness is the reality that interpersonal interactions, both in-person and online, have separate positive and negative dimensions (Kent de Grey et al., 2019). In adults, a study that investigated both facets of online interactions found that positive online experiences, related to 
ADOLESCENT LONELINESS AND ONLINE SOCIAL EXPERIENCES IN COVID-19

"capitalization, emotional, informational, and belonging social support" (Kent de Grey et al., 2019, p. 2), were associated with cardiovascular health; however, negative online experiences including "exclusion, rejection, interference and stigmatization" were associated with cardiovascular problems (Kent de Grey et al., 2019, p. 2). To date, these separable dimensions of online interpersonal interactions have not been studied in adolescence.

\section{Cultural Considerations}

The values and meanings of interpersonal relationships are strongly influenced by culture (Chen \& French, 2007; van Staden \& Coetzee, 2010). The majority of cross-cultural studies of loneliness have focused mostly on European or North American populations (Chen et al., 2004; Stickley et al., 2014), finding little difference between different countries. However, a recent large-scale study across 237 countries and territories found loneliness was positively associated with individualistic countries (Barreto et al., 2020). Furthermore, other literature has found the reverse, with loneliness being negatively associated with individualism (Lykes \& Kemmelmeier, 2014; Yum, 2003). Research suggests that while in individualistic cultures the absence of interactions with friends may predict loneliness, the absence of family interactions may better predict loneliness in collectivistic cultures (Lykes \& Kemmelmeier, 2014). Thus, in collectivistic cultures like Perú's and the majority of Latin America's (Hofstede Insights, 2020), increased time with family may be especially protective against loneliness amidst interrupted friend and peer relationships during COVID-19.

In addition to the loneliness literature, the majority of research on the impact of digital media on adolescent wellbeing has also focused on populations of adolescents growing up in the Global North (Magis-Weinberg, 2021; Odgers \& Jensen, 2020; Orben, 2020). In Perú, internet penetration is increasing, particularly during the pandemic. In 2020, 85\% of Peruvians adolescents had internet access, albeit with persistent access gaps between urban and rural areas, and mostly through smartphones and not computers (Carhuavilca Bonett et al., 2020). Social media is a very popular activity among Peruvian adolescents, and Facebook, WhatsApp and Instagram are the dominating platforms (Statista, 2019). Digital divides in use and appropriation, which intersect with gender norms and barriers, continue to influence online experiences for youth in low- and middle-income countries (Magis-Weinberg, Suleiman \& Dahl, 2021). Furthermore, there is emerging evidence from work with young adults in collectivistic cultures for tighter and closer social media networks than in individualistic settings ( $\mathrm{Na}$ et al., 2015). 


\section{ADOLESCENT LONELINESS AND ONLINE SOCIAL EXPERIENCES IN COVID-19}

In the face of a globalized pandemic, it is important to characterize how youth in different settings are addressing the many challenges presented by this situation. Here we focus on adolescents in Perú whose government imposed very stringent stay at home orders for the first 100 days compared to other countries in Latin America: children could not move between the homes of separated parents, meet friends in outdoor spaces, or more broadly leave the house.

\section{Current Study}

In a longitudinal, observational study following adolescents from low-to-middle income urban settings, we investigate whether perceived positive or negative online experiences relate to loneliness during five weeks of lockdown in the early stages of the COVID-19 pandemic in Perú. We pre-registered our hypotheses that positive online experiences would be related to lower feelings of loneliness, with an opposite pattern for negative experiences, and that these experiences would moderate the change in loneliness between lockdown week 6 and week 11 (https://osf.io/md36g). We investigated cross-sectional and longitudinal associations between adolescents' online positive and negative social experiences and loneliness during lockdown. The robustness of the association was tested by controlling for screen time and perceived family and friend social support. This study characterizes the association between online experiences and loneliness of adolescents in an increasingly virtually connected social contexts in the absence of in-person connections due to the COVID-19 lockdown.

\section{Participants and Procedure}

\section{Method}

Data were collected from April to June 2020 (sixth to eleventh week of lockdown; Figure 1) as part of the administration of a six-week well-being curriculum designed to promote wellbeing and healthy technology use among Peruvian students during the COVID-19 lockdown (Magis-Weinberg, Berger, Gys \& Dahl, 2021; Magis-Weinberg, 2021). Of the original 3,859 students who participated in the curriculum, 735 completed the surveys with measures relevant to the current study. The rest completed different surveys with other measures that are reported elsewhere (Magis-Weinberg, Berger, Gys \& Dahl, 2021).

This final sample were between sixth and eleventh grade (63.2\% female), were on average 13.3 years old (range $=11.2$ - 17.2) and were from 63 campuses of a low-cost private school system in Perú that is designed to support students of emerging middle-class families in Latin America. 
ADOLESCENT LONELINESS AND ONLINE SOCIAL EXPERIENCES IN COVID-19

All measures were delivered remotely via Qualtrics during the advisory period of the regular school schedule at different time points (see Figure 1 for a detailed timeline). After providing demographic information, participants completed a battery of self-report surveys on social connectedness, mental health, and digital media use. Ethics approval was granted by the university human subjects committee. 


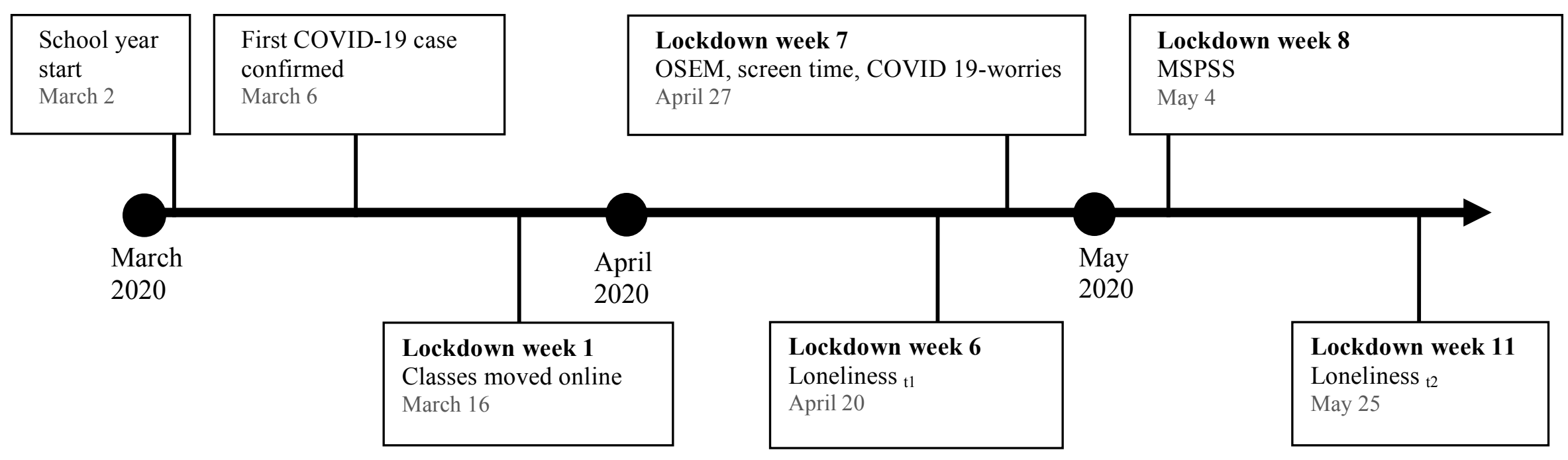

Figure 1. Timeline displaying important dates relevant to students' academic calendar, COVID-19 in Perú, and data collection for the current study expressed in weeks since start of lockdown. Note that there are no measurements before the lockdown. 
ADOLESCENT LONELINESS AND ONLINE SOCIAL EXPERIENCES IN COVID-19

\section{Measures}

A detailed description of all measures and English translations, as well as pre-registered hypotheses and analyses can be found in Open Science Framework (https://osf.io/md36g).

\section{Loneliness (lockdown weeks 6 and 11)}

Loneliness was the dependent variable and was assessed twice. Through an adapted nineitem version of the 10-item short form of the UCLA Loneliness Scale (Version 3; Russell, 1996), participants reported on the frequency of experiencing loneliness during the past two weeks on a four-point scale $(1=$ never, $4=$ always $)$. Though items are typically summed to yield a total score, we calculated the score by averaging items to make comparison with other studies possible. We excluded participants who were missing three or more items. The scale showed good reliability (Cronbach's alpha $=.79)$.

\section{Online Social Experiences Measure (lockdown week 7)}

Participants' experiences of social support and negativity online were assessed using an adapted version of the Online Social Experiences Measure (OSEM) scale (Kent de Grey et al., 2019). Divided into two subscales, OSEM measured the independent effects of positive and negative online social experiences with 11 items-five measuring positive experiences (e.g., "I feel valued by people in my social media", "People in my social media give me advice") and six measuring negative experiences (e.g. "People in my social media make me feel like I do not belong", "People in my social media treat me badly") — on a five-point scale assessed frequency $(1=$ never to $5=$ very frequent $)$ of experiences during the past month of COVID-19 lockdown. Mean scores for each subscale were calculated and used for analyses. We excluded participants missing two or more items on the subscale (positive or negative) being used in the analyses. The subscales showed good reliability (Cronbach's alpha $\mathrm{OSEM}_{\text {positive }}=.85$, Cronbach's alpha OSEM $_{\text {negative }}=.79$ ).

\section{Screen Time and Media Use (lockdown week 7)}

Participants' screen time was measured using four questions that asked for the number of hours spent on the following devices in a typical day of lockdown for non-academic purposes: television, video games, computers, and mobile devices. Participants could select responses ranging from zero hours to eight or more hours (in integers increasing by one). Rates of personal and family device ownership, popular platforms and accounts were assessed.

\section{COVID-19 worries (lockdown week 7)}




\section{ADOLESCENT LONELINESS AND ONLINE SOCIAL EXPERIENCES IN COVID-19}

Main sources of worry related to COVID-19 were assessed regarding 13 items: Staying at home, Not seeing friends in person, Getting sick, Family getting sick, Friends getting sick, Getting behind in school, Spending more time with family, Parents losing their job, Money, Conflicts with siblings, Conflicts with parents, Missing milestones, Getting into university. Participants endorsed their level of worry on a three-point scale $(1=$ not at all, $2=a$ little, $3=a$ lot).

\section{Multidimensional Scale of Perceived Social Support (lockdown week 8)}

The Multidimensional Scale of Perceived Social Support (MSPSS; Zimet, 2016) was administered to measure participants' perceived social support during the preceding week from three sources: family, friends, and a significant other. MSPSS consists of 12 items that participants endorsed on a seven-point scale $(1=$ strongly agree, $7=$ strongly disagree $)$, with higher scores indicating more perceived support. Item responses were averaged to find the score for each subscale, and subscale scores were summed to find the total score. In analyses using the MSPSS total scale, we excluded those missing four items or more. For analyses using any of the MSPSS subscales, which contained four items each, we excluded participants missing two or more items. The subscales showed good reliability (Cronbach's alpha for MSPSS $_{\text {family }}=.88$, Cronbach's alpha for MSPSS $_{\text {friends }}=.90$, Cronbach's alpha for MSPSS $_{\text {significant other }}=.86$, Cronbach's alpha for MSPSS $\left._{\text {total }}=.90\right)$.

\section{Data Analyses}

All data analyses were conducted in R 4.0.2 (R Core Team, 2020). Descriptive analyses were done using apaTables to calculate mean and standard deviations and bivariate correlations between variables (Stanley \& Spence, 2018), and compareGroups for group comparisons (Subirana et al., 2014).

\section{Data Cleaning}

Out of the total 735 participants, outliers were defined as those values more than 1.5 times the interquartile range and were removed from further analyses from the following variables: OSEM positive ( $0 \%$ excluded), OSEM negative (5.2\% excluded), MPSS family $(0.8 \%$ excluded), MSPSS friends ( $0 \%$ excluded), and loneliness $(0.3 \%$ excluded). Analyses were conducted with and without outliers, with very similar patterns of results. Hence, results reported below correspond to analyses excluding outlier data. 


\section{ADOLESCENT LONELINESS AND ONLINE SOCIAL EXPERIENCES IN COVID-19}

\section{Hypothesis Testing}

The goal of this study was to investigate the change in self-reported loneliness between week six and week eleven of the pandemic, and to investigate how social positivity and negativity online are associated with loneliness measured at week seven. We tested our hypothesis using a mixed effects model comparison approach pre-registered at: https://osf.io/md36g. Loneliness was the dependent variable in all mixed effects models, which included participant ID as a random effect. Session was included in all models as a predictor to test for change between week six and week eleven. Gender and age were included to control for potential differences in loneliness. Family support, friend support, and screen time measured at week eight were included as covariates to control for in-person support and engagement with technology. To test whether magnitude or change of loneliness was different depending on the valence of online experiences, we tested for OSEM and session interactions. Finally, we tested for three-way interactions between gender or age, OSEM, and session. All models were fitted with full information maximum likelihood estimation. Model fitting was performed using R (R Core Team, 2020) and package lme4 (Bates et al., 2020). Models were compared based on the Akaike Information Criterion (AIC; Akaike, 1974), using log likelihood ratio tests for improvement of model fit for nested models. Model fit was assessed using conditional $\mathrm{R}^{2}$ for generalized linear mixed-effects models (Nakagawa et al., 2017). Pairwise comparisons employed Tukey's method for multiple tests.

Changes to pre-registered analyses include: (1) grade was replaced with age as a covariate for parsimony, (2) friend support was included as a covariate in the models in addition to family support, (3) a full model including both positive and negative experiences was also explored and has become the primary focus of this study.

\section{Results}

\section{Descriptive Data}

\section{Media use}

The vast majority of students (90\%) own a smartphone, and all families have at least one device. Mobile use was the preferred medium to connect to the internet for non-school purposes (50\%), followed by laptops (16\%) and videogame consoles (12\%). Mobile use was higher for

older students. The two most popular activities for girls were social media and messaging (70\%) and watching videos (e.g. Youtube, 66\%). For boys, social media and messaging were popular 
ADOLESCENT LONELINESS AND ONLINE SOCIAL EXPERIENCES IN COVID-19

(55\%), but less so than videogames (61\%) and watching videos (62\%). Social media was more popular for older students, with an opposite pattern for videogames.

\section{COVID-19 worries}

On week seven, in early stages of the COVID-19 pandemic, the main worries endorsed by participants related to family getting sick $(M=2.7, S D=0.6)$, followed by friends getting sick $(M=2.4, S D=0.7)$ and getting behind in school $(M=2.4, S D=0.7)$. Other frequent concerns were not seeing friends in person $(M=2.3, S D=0.8)$, economic issues such as parents losing their job $(M=2.2, S D=0.8)$, money worries $(M=2.2, S D=0.8)$, and getting sick $(M=2.1, S D$ $=0.9)$. The least endorsed concerns related to having to stay at home $(M=1.9, S D=0.9)$ and conflict with parents $(M=1.9, S D=0.8)$. Increased time with family was the lowest concern $(M$ $=1.6, S D=0.7)$.

\section{Loneliness, Online Experiences, Screen time and Family Support}

Descriptive statistics for main variables of interest are shown in Table 1. Correlations among variables are presented in Table 2. At both week six and week 11, males exhibited less loneliness than females. Though age was not correlated with loneliness at week six, older students endorsed higher rates of loneliness scores at week 11.

While both genders had comparable positive online experiences, males experienced fewer negative online experiences than females. Older students experienced positive and negative online experiences more frequently. Overall, reports of positive online experiences $(M=$ 3.1) were more frequent than reports of negative online experiences $(M=1.3)$, with up to $46 \%$ of the sample reporting never having negative online experiences. In the sample, $48 \%$ of participants reported having lower than the median frequency scores for both positive and negative online experiences, $41 \%$ of participants reported frequent positive but infrequent negative experiences, $7 \%$ of participants endorsed infrequent positive and frequent negative experiences, and $5 \%$ of the sample reported frequent positive and negative online experiences.

While males perceived more family support than females, both genders perceived similar levels of social support from friends. Older students perceived less family support and more friend social support. Males recorded significantly less screen time than female students. Screen time was positively associated with age. 
ADOLESCENT LONELINESS AND ONLINE SOCIAL EXPERIENCES IN COVID-19

Table 1

Descriptive Statistics of Study Variables Grouped by Gender

\begin{tabular}{|c|c|c|c|c|c|c|}
\hline & & le & & & & \\
\hline & $M$ & $S D$ & $M$ & $S D$ & $t$-test & $p$ \\
\hline Age (years) & 13.4 & 1.50 & 13.1 & 1.43 & 2.469 & $.01^{*}$ \\
\hline $\begin{array}{l}\text { Loneliness } \\
\text { (Week 6) } \\
\text { (possible } \\
\text { range: } 1-4 \text { ) }\end{array}$ & 1.94 & 0.57 & 1.80 & 0.49 & 3.420 & $\begin{array}{c}< \\
.001^{* * *}\end{array}$ \\
\hline $\begin{array}{l}\text { Loneliness } \\
\text { (Week 11) } \\
\text { (possible } \\
\text { range: } 1-4 \text { ) }\end{array}$ & 1.95 & 0.61 & 1.83 & 0.54 & 2.680 & $.006 * *$ \\
\hline $\begin{array}{l}\text { OSEM positive } \\
\text { (possible } \\
\text { range: } 1-5 \text { ) }\end{array}$ & 3.10 & 1.06 & 3.00 & 1.10 & 1.157 & .25 \\
\hline $\begin{array}{l}\text { OSEM } \\
\text { negative } \\
\text { (possible } \\
\text { range: } 1-5 \text { ) }\end{array}$ & 1.28 & 0.34 & 1.22 & 0.30 & 2.288 & $.02 *$ \\
\hline $\begin{array}{l}\text { MSPSS-family } \\
\text { (possible } \\
\text { range: } 0-6 \text { ) }\end{array}$ & 5.32 & 1.42 & 5.73 & 1.29 & -3.622 & $<.001 * * *$ \\
\hline $\begin{array}{l}\text { MSPSS-friends } \\
\text { (possible } \\
\text { range: } 0-6 \text { ) }\end{array}$ & 5.40 & 1.49 & 5.19 & 1.54 & 1.680 & 0.10 \\
\hline $\begin{array}{l}\text { Screen time } \\
\text { (possible } \\
\text { range: } 0-8+\mathrm{h} \text { ) }\end{array}$ & 6.21 & 4.00 & 4.92 & 3.52 & 4.367 & $<.001 * * *$ \\
\hline
\end{tabular}


ADOLESCENT LONELINESS AND ONLINE SOCIAL EXPERIENCES IN COVID-19

Note. $M=$ mean, $S D=$ standard deviation. ${ }^{*} p<0.05,{ }^{*} p<0.01$ and $* * * p<0.001$. OSEM $=$ online social experience measure, MSPSS = multidimensional scale of perceived social support. 


\section{ADOLESCENT LONELINESS AND ONLINE SOCIAL EXPERIENCES IN COVID-19}

Screen time was positively correlated with both positive and negative online experiences, highlighting the need to control for screen time in analyses. Participants' screen time on mobile devices $(r(714)=0.16, p<.0001)$ was more strongly correlated with their positive online experiences than their time on television $(r(715)=0.16, p=.30)$ and video games $(r(712)=-.00$, $p=.97)$. The same pattern was found for negative online experiences, with higher correlations for screen time on computers $(r(715)=.09, p=.01)$ and mobile devices $(r(714)=.20, p<.0001)$ than television $(r(715)=-.03, p=.40)$ and video games $(r(712)=.01, p=.90)$, perhaps due to the greater range of social platforms on these former devices. Thus, contrary to our pre-registered analytical plan, hours per typical day of lockdown on only computers and mobile devices were summed to yield the screen time measure used in analyses.

Family support showed the weakest correlation with positive online social experiences, suggesting they capture distinct forms of support. However, total support, friend support, and significant other support were most strongly associated with positive online experiences, suggesting overlapping sources of social support presumably due to the substitution of in-person interactions with online ones during COVID-19. Total support and family support were negatively correlated with negative online experiences. Screen time was negatively associated with perceived family support and positively correlated with perceived friend support, adding evidence to the offline and online sources of support for adolescents at this time.

We included MSPSS as a robust and often used measure of in-person family, friend and significant other support. Due to the nature of social isolation, the interpretation of items of MSPSS friends in particular was likely reflective of online support as well. In a departure from pre-registered analyses, we decided to include friend support as a covariate in analyses to see if general online interactions predicted loneliness above and beyond general support from friends. Given the correlation between MSPSS friends and OSEM positive ( $\mathrm{r}=.45, p<.01)$, we chose to use MSPSS family as a proxy of in-person support during isolation in the mixed effects models. We omitted MSPSS significant other from the models due to the ambiguity of the language, especially for younger participants. 
ADOLESCENT LONELINESS AND ONLINE SOCIAL EXPERIENCES IN COVID-19

\section{Table 2}

Means, Standard Deviations, and Correlations with Confidence Intervals

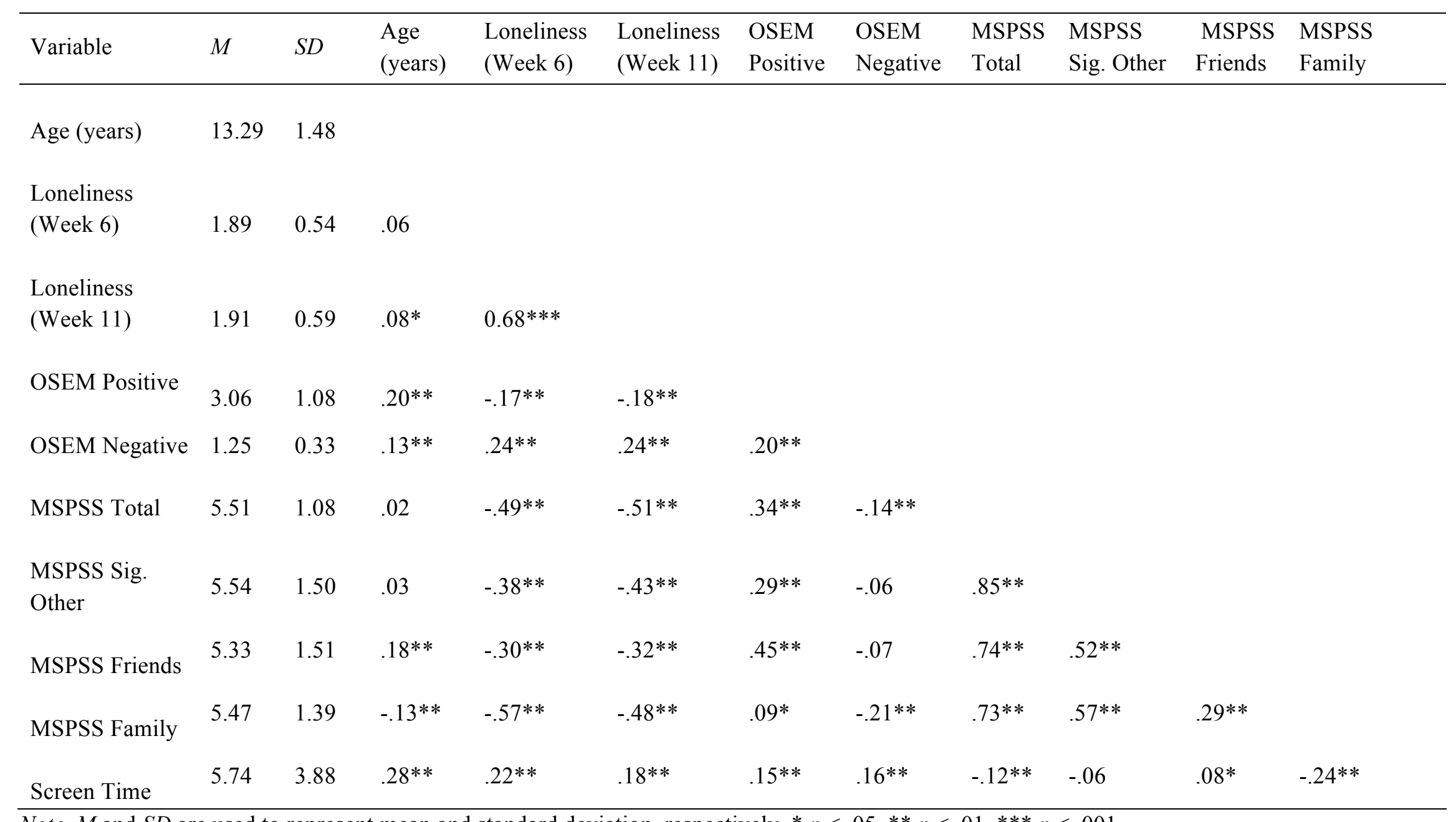

Note. $M$ and $S D$ are used to represent mean and standard deviation, respectively. ${ }^{*} p<.05,{ }^{* *} p<.01, * * * p<.001$ 
ADOLESCENT LONELINESS AND ONLINE SOCIAL EXPERIENCES IN COVID-19

\section{Main effects model}

Using a model comparison approach, we sequentially compared seven global models (Table 3). 
ADOLESCENT LONELINESS AND ONLINE SOCIAL EXPERIENCES IN COVID-19

Table 3

Linear Mixed Effects Models Predicting Loneliness.

\begin{tabular}{|c|c|c|c|}
\hline \multicolumn{2}{|r|}{$\begin{array}{c}\text { Models } \\
\text { (DV: Loneliness) }\end{array}$} & AIC & $\begin{array}{l}\text { Log likelihood } \\
\quad(p \text {-value })\end{array}$ \\
\hline Demographics & $\begin{array}{l}\text { Loneliness } \sim \text { session }+ \text { gender }+ \text { age }+ \\
(1 \mid \text { ID) }\end{array}$ & 1714 & -850.995 \\
\hline $\begin{array}{l}\text { Demographics } \\
\text { and controls }\end{array}$ & $\begin{array}{l}\text { Loneliness } \sim \text { session }+ \text { age }+ \text { screen time } \\
+ \text { family social support }+ \text { friend social } \\
\text { support }+(1 \mid \text { ID })\end{array}$ & 1382 & $\begin{array}{l}-681.99 \\
(p<.001)\end{array}$ \\
\hline $\begin{array}{l}\text { Online social } \\
\text { experiences }\end{array}$ & $\begin{array}{l}\text { Loneliness } \sim \text { session }+ \text { online } \\
\text { experiences }+ \text { gender }+ \text { age }+ \text { screen } \\
\text { time }+ \text { family social support }+ \text { friend } \\
\text { social support }+(1 \mid \text { ID })\end{array}$ & 1257.8 & $\begin{array}{c}-617.88 \\
(p<.001 * * *)\end{array}$ \\
\hline $\begin{array}{l}\text { Full } \\
\text { interaction }\end{array}$ & $\begin{array}{l}\text { Loneliness } \sim \text { session }+ \text { positive online } \mathrm{x} \\
\text { negative online }+ \text { age }+ \text { gender }+ \text { screen } \\
\text { time }+ \text { family social support }+ \text { friend } \\
\text { social support }+(1 \mid \text { ID })\end{array}$ & 1259.0 & $\begin{array}{l}-617.52 \\
(p=.40)\end{array}$ \\
\hline $\begin{array}{l}\text { Interactions } \\
\text { between online } \\
\text { social } \\
\text { experiences } \\
\text { and session }\end{array}$ & $\begin{array}{l}\text { Loneliness } \sim \text { session } x \text { positive online } \\
\text { experiences }+ \text { negative online } \\
\text { experiences }+ \text { gender }+ \text { age }+ \text { screen time } \\
+ \text { family social support }+ \text { friend social } \\
\text { support }+(1 \mid \text { ID })\end{array}$ & 1259.5 & $\begin{array}{l}-614.76 \\
(p=.14)\end{array}$ \\
\hline $\begin{array}{l}\text { Four-way } \\
\text { interaction } \\
\text { with gender }\end{array}$ & $\begin{array}{l}\text { Loneliness } \sim \text { session } x \text { positive online } \\
\text { experiences } x \text { negative online experiences } \\
\mathrm{x} \text { gender }+ \text { age }+ \text { screen time }+ \text { family } \\
\text { social support }+ \text { friend social support }+ \\
(1 \mid \text { ID) }\end{array}$ & 1271.5 & $\begin{array}{l}-613.78 \\
(p=.96)\end{array}$ \\
\hline $\begin{array}{l}\text { Four-way } \\
\text { interaction } \\
\text { with age }\end{array}$ & $\begin{array}{l}\text { Loneliness } \sim \text { session } x \text { positive online } \\
\text { experiences } x \text { negative online experiences } \\
x \text { age }+ \text { gender }+ \text { screen time }+ \text { family } \\
\text { social support }+ \text { friend social support }+ \\
(1 \mid \text { ID) }\end{array}$ & 1266.8 & $\begin{array}{c}-611.37 \\
(\mathrm{p}<.001 * * *)\end{array}$ \\
\hline
\end{tabular}

Note. Preferred model (in bold) was selected based on AIC values by sequentially comparing nested models. Models with interaction effects also included a main effect for these factors. AIC $=$ Akaike information criterion, ${ }^{*} p<0.05, * * p<0.01, * * * p<0.001$ 
ADOLESCENT LONELINESS AND ONLINE SOCIAL EXPERIENCES IN COVID-19

The best fitting model (conditional $\mathrm{R}^{2}=0.66$ ) revealed main effects of both positive and negative online experiences on loneliness at week six and week eleven, controlling for family social support, friend social support, gender, and age (Table 4). More negative online experiences were associated with greater loneliness, and more positive experiences were associated with less loneliness. Males reported less loneliness than females. Both higher family and friend support were associated with less loneliness. Lastly, screen time was positively associated with loneliness. Session and age were not significantly related to loneliness. The model testing their main effects yielded better fit than the models assessing their interactive effects

The pattern of significance was similar when positive and negative online experiences were analyzed separately. Positive online experiences were related to less loneliness $(\beta=-.08$, $95 \% C I[-0.14,-0.01], p=.02)$, and negative ones were related to more loneliness $(\beta=.16,95 \%$ CI [ $0.10,0.22], \mathrm{p}<0.001)$. Interactions with session were not significant in either case. 


\section{ADOLESCENT LONELINESS AND ONLINE SOCIAL EXPERIENCES IN COVID-19}

Table 4

Parameter Estimates for the Best Fitting Model including both OSEM Positive and Negative as

Predictors.

\section{Loneliness (outcome)}

\begin{tabular}{lcccc}
\hline \multicolumn{1}{c}{ Fixed effects } & $\beta$ & $95 \%$ CI & $\mathrm{t}$ & $p$ \\
& \multicolumn{1}{l}{ [LL, UL] } & & \\
& -.09 & {$[-0.19,0.01]$} & 18.23 & $<.001 * * *$ \\
\hline Intercept & .03 & {$[-0.04,0.11]$} & 0.85 & .40 \\
Session & -.14 & {$[-0.20,-0.07]$} & -4.21 & $<.001 * * *$ \\
OSEM Positive & .19 & {$[0.13,0.25]$} & 6.5 & $<.001 * * *$ \\
OSEM Negative & -.12 & {$[-0.23,0.00]$} & -2.04 & $.04 *$ \\
Gender (Males) & -.01 & {$[-0.06,0.05]$} & -0.18 & .86 \\
Age (Years) & .09 & {$[0.03,0.15]$} & 3.12 & $<.01 * *$ \\
Screen time & -.43 & {$[-0.49,-0.37]$} & -14.00 & $<.001 * * *$ \\
MSPSS Family & -.14 & {$[-0.21,-0.08]$} & -4.35 & $<.001 * * *$
\end{tabular}

Note. Females served as the reference level for gender. OSEM $=$ online social experience measure, MSPSS $=$ multidimensional scale of perceived social support, $\beta=$ standardized coefficient, $\mathrm{CI}=$ confidence interval, $\mathrm{LL}=$ lower limit, $\mathrm{UL}=$ upper limit. ${ }^{*} p<0.05,{ }^{* *} p<0.01, * * * p<0.001$ 
ADOLESCENT LONELINESS AND ONLINE SOCIAL EXPERIENCES IN COVID-19

\section{Discussion}

The aim of this study was to examine levels of loneliness during early stages of physical isolation in response to the COVID-19 pandemic in adolescents in Perú. Further, we investigated whether loneliness was associated with online experiences, an important source of peer connection at a time of remote instruction. We found that loneliness did not change from week six to week 11 of lockdown, was higher for females, and similar across ages during week 6 with some evidence for an increase with age during week 11. Perceptions of positive online experiences were more frequent than negative experiences, with both online experiences reported as more frequent for older students. Females experienced more negative online experiences than males. Lower loneliness was associated with lower negative experiences and also higher positive experiences. These associations held when positive and negative experiences were separated into different models and also when analyzed together in the same model, when controlling for inperson family social support, friend social support, and screen time. Our results highlight the association between online social support and negativity and loneliness, suggesting how positive online experiences may be an important vehicle for social connection in the face of lockdown.

\section{Perceived Online Positivity and Negativity and Loneliness}

Adolescents' self-report of their online experiences was predominantly positive. The majority of participants reported very infrequent negative online experiences in combination with either infrequent positive experiences or frequent positive experiences. A primary finding of our study is that youths' engagement with their online community differentially associates with loneliness, depending on the nature of these interactions, separating the positive and negative dimensions of interpersonal relationships online (Kent de Grey et al., 2019). OSEM positive items capture social support, while OSEM negative items capture rejection (Kent de Grey et al., 2019). That is, adolescents reporting greater positive online social experiences (including emotional and informational support and belonging; Kent de Grey et al., 2019) endorsed lower levels of loneliness. This finding on the importance of social support is consistent with a recent study linking low-to-medium social support during COVID-19 with increased symptoms for depression and anxiety in a sample of Chinese adolescents (Qi et al., 2020). In contrast, when online interactions with peers were negative (e.g., perceived exclusion), adolescents reported greater feelings of loneliness. Alternatively, it may be that perceived loneliness influences the perception of online experiences or the likelihood of engaging in positive or negative interactions 
ADOLESCENT LONELINESS AND ONLINE SOCIAL EXPERIENCES IN COVID-19

online, such that those who are lonelier either perceive or tend to engage in fewer positive experiences and greater negative experiences.

Also noteworthy is that the effect of OSEM negative was only slightly stronger than the effect of OSEM positive. In contrast to previous research (Primack et al., 2019), we do not find strong evidence that negative experiences outweigh the association of positive experiences with loneliness. Thus, it appears it may be equally important to both reduce negative social experiences and promote positive ones online in treatments, interventions, and policies aiming to mitigate loneliness.

Taken together, the nature of adolescents' interactions with peers online is critical for investigating how time spent online may promote or detract from adolescent loneliness and wellbeing, or how levels of loneliness may influence the perceived nature of online interactions.

Previous mixed findings on the relationship between loneliness and online interactions might be partially explained by a narrow focus on either the negative or positive dimensions of digital media that are studied. It is important to investigate, at the same time, and in the same sample, both positive and negative separable dimensions of online interactions to increase our understanding of digital media's impact on well-being. Our results align with the interpersonalconnection-behaviors framework (Clark et al., 2018), showing that the type of interaction online matters for the well-being outcome in question. Similarly, these results are consistent with Kross et al.'s (2020) literature review on the differential links between social media use and well-being when considering how media is used. Lastly, while we did not explicitly measure objective social isolation from peers, it is reasonable to assume that sibling interactions constituted the main - or more likely only — potential peer contact of the current sample given the strictness of the lockdown in Perú. Thus, we speculate that online interactions have become even more important during COVID-19 since communicating online was the only vehicle to chat with friends.

\section{Screen Time}

All participants of this study had access to either their own or a family device and everyone had access to the internet at home. While large and diverse, our sample is not representative of the country, which still faces important digital divides. In the current study, participants reported 5.6 hours of recreational screen time in April 2020. This represents an increase when compared to findings from a different sample from the same school context who 
ADOLESCENT LONELINESS AND ONLINE SOCIAL EXPERIENCES IN COVID-19

reported 2.5 hours of recreational screen time in 2019. These results are in line with national trends reporting an increase in internet use for Peruvian youth from $77.4 \%$ in 2019 to $85.7 \%$ in 2020 (Carhuavilca Bonett et al., 2020).

Prior research utilizing crude measures of screen time have found mixed evidence regarding social media and loneliness (see Kross et al., 2020 for a synthesis of prior research and weaknesses of prior social media research using aggregate social media usage). In this study, we find some evidence for increased recreational screen time compared to before the pandemic, and that older adolescents reported higher levels of screen time. As screen time surges, the frequency of both negative and positive experiences will likely increase. While past research has highlighted the need to characterize online activity as active or passive to adequately understand its relation to mental health outcomes (Burke \& Kraut, 2016), the current study highlights another important qualifier: the positive or negative nature of online interactions. Thus, our findings contribute to a growing body of evidence suggesting that quality of interactions and motivations for social media use are more important than quantity of social media use in determining potential impacts on well-being (Davila et al., 2012; Kross et al., 2020).

Based on our study (and recent practice recommendations: Domoff, 2021), it is critical to clarify the nature of peer interactions online. For some adolescents, positive online social interactions may mitigate the temporary loss of in-person interactions with peers. Given the restrictions on socially-intimate interactions during the pandemic, parents, educators, and clinicians may seek to promote increased interactions with peers online. However, clinicians working with youth who are reporting increased loneliness during the pandemic should assess for the quality of online social interactions and offer strategies for improving online social skills and adaptive engagement with peers (e.g., Domoff, under review; Domoff, Borgen \& Robinson, 2020). Adolescents with strong offline connections may benefit more from online interactions (Lee, 2009), whereas those who already struggle with offline social skills may be more likely to experience poorer online social interactions (George \& Odgers, 2015). As such, youth who are most vulnerable may likely need support to increase positive online social interactions during times of heightened isolation. Promoting positive online experiences might be a fruitful avenue for intervention, as lonely individuals turn to online tools for relationship management (Morahan-Martin \& Schumacher, 2003). 
ADOLESCENT LONELINESS AND ONLINE SOCIAL EXPERIENCES IN COVID-19

We unexpectedly found that loneliness levels did not change over the six weeks of this study. It is possible that assessing loneliness over a longer duration (e.g., several months of lockdown) would have provided a wider range in loneliness change scores. On the other hand, it is important to note that the association between perceived social support and loneliness was strongest when considering social support from family (e.g., parents, siblings), versus social support from friends. Thus, it is possible that with the nature of the lockdown, youth had more contact with family members, which may have mitigated an increase in loneliness. Indeed, increased family time was identified as the main positive outcome of the lockdown by the majority of the adolescents in the study. In a collectivistic culture like Perú (Hofstede Insights, 2020), family support may be especially protective against loneliness. Like past studies, collectivistic cultural values may also have engendered more tight-knit social media networks for adolescents mitigating increases in loneliness (Cho et al., 2013; Na et al., 2015). Future research on the impact of the pandemic on loneliness should continue to consider the role of family social support and cultural values in stabilizing or promoting adolescent well-being during lockdown periods.

\section{Loneliness and Social Support}

In line with previous literature (von Soest et al., 2020), we found that females reported greater loneliness compared to males in this sample at week six and week 11. At week six, we did not find age differences on loneliness, which aligns with previous studies (Ellis et al., 2020; von Soest et al., 2020). However, some age differences emerge five weeks later, with older students exhibiting higher levels of loneliness than younger students as the lockdown progressed. Emerging findings on the impact of COVID-19 on adolescent well-being are pointing towards a particular vulnerability of older adolescents (Smirni et al., 2020; Zhou et al., 2020). Longitudinal follow-ups should explore whether age differences manifested as the lockdown progressed.

We do not have pre-COVID data on this sample, and, to our knowledge, there are no normative reports of UCLA loneliness in Perú. However, compared to samples in other countries, it would appear that current results are in line with pre-COVID levels of loneliness in adolescence (Alpaslan et al., 2016; Mahon et al., 2001; Neto \& Barros, 2000; Purwono \& French, 2016). While physical isolation may prevent in-person interaction, tech-savvy adolescents may still be able to meet their social connectivity needs by bridging the physical gap through social media. Family is also an important source of social support and might be a 
ADOLESCENT LONELINESS AND ONLINE SOCIAL EXPERIENCES IN COVID-19

protective factor. In fact, in our analyses, family support is more strongly associated with loneliness than friend support. While we do not have objective data for COVID-19 impacts in the early stages of the pandemic for these youth, self-reported concern around family or friends getting sick were the most endorsed items. In contrast, having to stay at home or increased time with family were the lowest ranking concerns. These findings align with the importance of family for these youth, and with how social support, especially from family, was associated with less loneliness.

As we predicted, family social support decreased with age, and an opposite pattern was observed for friend social support, in line with reported trends (Helsen et al., 2000). The social reorientation in adolescence (Nelson et al., 2015) has been well documented, with a departure from parents and reorientation towards friends and peers. While in pre-COVID times, objective time with parents decreases during adolescence, as they choose to spend more time with their peers, during lockdown, adolescents are objectively spending much more time with parents. Still, as a reflection of a subjective perception of (vs. objective) sources of social support, older ages report less family support than younger grades.

\section{Limitations and Future Directions}

Strengths of this study include a large, diverse sample of Peruvian adolescents, who are traditionally under-represented on research on screen time and adolescent well-being (Odgers \& Jensen, 2020). Additionally, methodological strengths include pre-registered and longitudinal analyses. Despite these strengths, there are some limitations to be addressed in future research. While the study was conducted during the early stages of the COVID-19 pandemic, and we documented some effects on adolescent worries, especially about the health of friends and family, and not being able to see friends in person, our design does not allow us to investigate the direct impact of the lockdown on the association between online experiences and loneliness. We can only speculate that online interactions became a much more important source of connection with friends, given physical isolation and remote instruction.

Only two time points of loneliness were collected (with a short duration between data collection points). It should be noted that these are self-reported by the adolescents themselves, in this way reflecting perceptions that youth might have of social isolation, sources of family and online support. As such, existing levels of loneliness might have biased how youth rated their social connectedness. Future research should include multiple time points and a greater duration 
ADOLESCENT LONELINESS AND ONLINE SOCIAL EXPERIENCES IN COVID-19

of time between data collection. This will be especially important in order to explore temporality and to elucidate the association between online social experiences and loneliness. Additionally, utilizing objective measures of adolescents' online interactions and screen time would address the limitation of using self-report for the measurement of study variables. Future studies should incorporate more specific measures of online behaviors. We used MSPSS as a proxy for youth perception on family support. However, we do not have data on the variance in household environment (e.g., parents' professions, economic hardship, COVID-19-related loss), which may influence perceived family support and other family dynamics during lockdown. Lastly, it should also be noted that data collection coincided with a light-touch intervention (Author, in press) aimed at providing basic tools and strategies to help participants cope with the transition to remote learning and quarantine. Due to the relatively short and non-intensive nature of the intervention, however, it is unlikely that it could have had a strong effect on loneliness.

\section{Conclusion}

Adolescence is a developmental stage marked by increased importance of social networks outside the family (e.g., peer relationships). The stay-at-home measures in response to the COVID-19 pandemic have intensified the digitization of social life, with online interactions almost completely replacing in-person interactions with peers. Answering the recent call for more specific measures of digital media use to understand its unique connections to adolescent well-being, and the importance of showcasing the experiences of underrepresented global youth, the current study assessed the differential association of positive and negative online experiences and loneliness in Peruvian adolescents from week six to week 11 of lockdown. Our results show that considering the nature of online experiences rather than more general measures of online use (e.g., screen time) is necessary for a more complete understanding of the links between digital media use and loneliness among adolescents. Youth perceive their positive online experiences to be much more frequent than negative online experiences. The findings that positive online experiences negatively associated with loneliness and negative online experiences positively associated with loneliness, above and beyond in-person social support from family and friends, highlight how positive digital media use may be leveraged to mitigate adolescent loneliness. Future research could elucidate the long-term effects of online social experiences and adolescent well-being more broadly during lockdown by assessing these relationships at multiple time points across a longer period of time. 


\section{References}

Akaike, H. (1974). A new look at the statistical model identification. IEEE Transactions on Automatic Control, 19(6), 716-723. https://doi.org/10.1109/TAC.1974.1100705

Allen, K. A., Ryan, T., Gray, D. L., McInerney, D. M., \& Waters, L. (2014). Social media use and social connectedness in adolescents: The positives and the potential pitfalls. The Australian Educational and Developmental Psychologist, 31(1), 18-31. https://doi.org/10.1017/edp.2014.2

Alpaslan, A. H., Kocak, U., \& Avci, K. (2016). Gender-Related Factors for Depressive Symptoms in Turkish Adolescents. Journal of Child and Adolescent Psychiatric Nursing, 29(1), 23-29. https://doi.org/10.1111/jcap.12131

B. Bradford Brown. (1990). Peer Groups and Peer Cultures. In At the Threshold: The Developing Adolescent (p. 24). Harvard University Press. https://prsg.education.wisc.edu/wpcontent/uploads/2014/08/Browqn-1990-Peer-groups-peer-cultures.pdf

B. Bradford Brown. (2004). Adolescents' Relationships with Peers. In Handbook of Adolescent Psychology (Second Edition, p. 25). John Wiley \& Sons, Inc.

https://prsg.education.wisc.edu/wp-content/uploads/2014/08/Brown-2004-Adol-relations-wpeers.pdf

Barreto, M., Victor, C., Hammond, C., Eccles, A., Richins, M. T., \& Qualter, P. (2020). Loneliness around the world: Age, gender, and cultural differences in loneliness. Personality and Individual Differences, 110066. https://doi.org/10.1016/j.paid.2020.110066

Bates, D., Maechler, M., Bolker, B., \& walker, S. (2020). Ime4: Linear Mixed-Effects Models using "Eigen" and S4 (1.1-26) [Computer software]. https://cran.rproject.org/web/packages/lme4/index.html

Berkman, L. F. (1995). The Role of Social Relations in Health Promotion. Psychosomatic Medicine, 57(3), 245-254.

Best, P., Manktelow, R., \& Taylor, B. (2014). Online communication, social media and adolescent wellbeing: A systematic narrative review. Children and Youth Services Review, 41, 27-36. https://doi.org/10.1016/j.childyouth.2014.03.001 
ADOLESCENT LONELINESS AND ONLINE SOCIAL EXPERIENCES IN COVID-19

Blakemore, S.-J., \& Mills, K. L. (2014). Is adolescence a sensitive period for sociocultural processing? Annual Review of Psychology, 65, 187-207. https://doi.org/10.1146/annurevpsych-010213-115202

Burke, M., \& Kraut, R. E. (2016). The Relationship Between Facebook Use and Well-Being Depends on Communication Type and Tie Strength. Journal of Computer-Mediated Communication, 21(4), 265-281. https://doi.org/10.1111/jcc4.12162

Carhuavilca Bonett, D. C., Sánchez Aguilar, A., Hidalgo Calle, N., Gutiérrez Espino, C., Mendoza Loyola, D., \& Ruiz Calderón, R. (2020). Estadísticas de las Tecnologías de Información y Comunicación en los Hogares: Julio-Agosto-Septiembre 2019-2020. (p. 55). Instituto Nacional de Estadística e Informática. https://www.inei.gob.pe/media/MenuRecursivo/boletines/04-informe-tecnico-tic-iiitrimestre2020.pdf

Carpenter, J. M., Green, M. C., \& LaFlam, J. (2011). People or profiles: Individual differences in online social networking use. Personality and Individual Differences, 50(5), 538-541. https://doi.org/10.1016/j.paid.2010.11.006

Cauce, A. M., Reid, M., Landesman, S., \& Gonzales, N. (1990). Social support in young children: Measurement, structure, and behavioral impact. In Social support: An interactional view (pp. 64-94). John Wiley \& Sons.

Chen, X., \& French, D. C. (2007). Children's Social Competence in Cultural Context. Annual Review of Psychology, 59(1), 591-616. https://doi.org/10.1146/annurev.psych.59.103006.093606

Chen, X., He, Y., Oliveira, A., Lo Coco, A., Zappulla, C., Kaspar, V., Schneider, B., Alvarez Valdivia, I., Tse, H., \& Desouza, A. (2004). Loneliness and social adaptation in Brazilian, Canadian, Chinese and Italian children: A multi-national comparative study. Journal of Child Psychology and Psychiatry, and Allied Disciplines, 45, 1373-1384. https://doi.org/10.1111/j.1469-7610.2004.00844.x

Cho, S. E., Jung, K., \& Park, H. W. (2013). Social Media Use during Japan's 2011 Earthquake: How Twitter Transforms the Locus of Crisis Communication. Media International Australia, 149(1), 28-40. https://doi.org/10.1177/1329878X1314900105

Choukas-Bradley, S., \& Prinstein, M. J. (2014). Peer Relationships and the Development of Psychopathology. In M. Lewis \& K. D. Rudolph (Eds.), Handbook of Developmental 
ADOLESCENT LONELINESS AND ONLINE SOCIAL EXPERIENCES IN COVID-19

Psychopathology (pp. 185-204). Springer US. https://doi.org/10.1007/978-1-4614-9608310

Chu, P. S., Saucier, D. A., \& Hafner, E. (2010). Meta-analysis of the relationships between social support and well-being in children and adolescents. Journal of Social and Clinical Psychology, 29(6), 624-645. https://doi.org/10.1521/jscp.2010.29.6.624

Clark, J. L., Algoe, S. B., \& Green, M. C. (2018). Social Network Sites and Well-Being: The Role of Social Connection. Current Directions in Psychological Science, 27(1), 32-37. https://doi.org/10.1177/0963721417730833

Colarossi, L. G., \& Eccles, J. S. (2003). Differential effects of support providers on adolescents' mental health. Social Work Research, 27(1), 19-30. https://doi.org/10.1093/swr/27.1.19

Dahl, R. E., Allen, N. B., Wilbrecht, L., \& Suleiman, A. B. (2018). Importance of investing in adolescence from a developmental science perspective. Nature, 554(7693), 441-450. https://doi.org/10.1038/nature25770

Davila, J., Hershenberg, R., Feinstein, B. A., Gorman, K., Bhatia, V., \& Starr, L. R. (2012). Frequency and Quality of Social Networking Among Young Adults: Associations With Depressive Symptoms, Rumination, and Corumination. Psychology of Popular Media Culture, 1(2), 72-86. https://doi.org/10.1037/a0027512

Davis, K. (2012). Friendship 2.0: Adolescents' experiences of belonging and self-disclosure online. Journal of Adolescence, 35(6), 1527-1536. https://doi.org/10.1016/j.adolescence.2012.02.013

Demaray, M. K., \& Malecki, C. K. (2002). The relationship between perceived social support and maladjustment for students at risk. Psychology in the Schools, 39(3), 305-316. https://doi.org/10.1002/pits.10018

Dienlin, T., Masur, P. K., \& Trepte, S. (2017). Reinforcement or Displacement? The Reciprocity of FTF, IM, and SNS Communication and Their Effects on Loneliness and Life Satisfaction. Journal of Computer-Mediated Communication, 22(2), 71-87. https://doi.org/10.1111/jcc4.12183

Dodge, K., \& Albert, D. (2012). Evolving Science in Adolescence. Developmental Psychology, 48(3), 624-627. https://doi.org/10.1037/a0027683

Domoff, S., Borgen, A., \& Robinson, C. (2020). Problematic use of screen media and mobile devices (pp. 175-198). https://doi.org/10.1016/B978-0-12-816024-4.00008-5 
ADOLESCENT LONELINESS AND ONLINE SOCIAL EXPERIENCES IN COVID-19

Domoff, S. (2021). Problematic Digital Media Use and Addiction. [Manuscript submitted for publication.]

Ellis, W. E., Dumas, T. M., \& Forbes, L. M. (2020). Physically isolated but socially connected: Psychological adjustment and stress among adolescents during the initial COVID-19 crisis. Canadian Journal of Behavioural Science / Revue Canadienne Des Sciences Du Comportement, 52(3), 177-187. https://doi.org/10.1037/cbs0000215

Ellison, N. B., \& Vitak, J. (2015). Social Network Site Affordances and Their Relationship to Social Capital Processes. In The Handbook of the Psychology of Communication Technology (pp. 203-227). John Wiley \& Sons, Ltd. https://doi.org/10.1002/9781118426456.ch9

Furman, W., \& Buhrmester, D. (1992). Age and Sex Differences in Perceptions of Networks of Personal Relationships. Child Development, 63(1), 103-115. https://doi.org/10.1111/j.14678624.1992.tb03599.x

Gardner, W. L., Pickett, C. L., \& Knowles, M. (2005). Social Snacking and Shielding: Using Social Symbols, Selves, and Surrogates in the Service of Belonging Needs. In The social outcast: Ostracism, social exclusion, rejection, and bullying (pp. 227-241). Psychology Press.

George, L. K., Blazer, D. G., Hughes, D. C., \& Fowler, N. (1989). Social Support and the Outcome of Major Depression. British Journal of Psychiatry, 154(4), 478-485. https://doi.org/10.1192/bjp.154.4.478

George, M. J., \& Odgers, C. L. (2015). Seven Fears and the Science of How Mobile Technologies May Be Influencing Adolescents in the Digital Age. Perspectives on Psychological Science, 10(6), 832-851. https://doi.org/10.1177/1745691615596788

Green, H., McGinnity, A., Meltzer, H., Ford, T., \& Goodman, R. (2005). Mental Health of Children and Young People in Great Britain, 2004: (557702010-001) [Data set]. American Psychological Association. https://doi.org/10.1037/e557702010-001

Hampton, K. N., Lu, W., \& Shin, I. (2016). Digital media and stress: The cost of caring 2.0. Information, Communication \& Society, 19(9), 1267-1286. https://doi.org/10.1080/1369118X.2016.1186714 
ADOLESCENT LONELINESS AND ONLINE SOCIAL EXPERIENCES IN COVID-19

Heinrich, L., \& Gullone, E. (2006). The clinical significance of loneliness: A literature review. Clinical Psychology Review, 26, 695-718. https://doi.org/10.1016/j.cpr.2006.04.002

Helsen, M., Vollebergh, W., \& Meeus, W. (2000). Social Support from Parents and Friends and Emotional Problems in Adolescence. Journal of Youth and Adolescence, 29(3), 319-335. https://doi.org/10.1023/A:1005147708827

Hoffart, A., Johnson, S. U., \& Ebrahimi, O. V. (2020). Loneliness and Social Distancing During the COVID-19 Pandemic: Risk Factors and Associations With Psychopathology. Frontiers in Psychiatry, 11. https://doi.org/10.3389/fpsyt.2020.589127

Hofstede Insights. (2020). Peru. Hofstede Insights: Consulting, Training, Certification, Tooling. https://www.hofstedeinsights.com/country/peru/\#: : :text=In\%20Individualist $\% 20$ societies\%20people\%20are,mos t\%20other\%20Latin\%20American\%20countries

Jose, P. E., \& Lim, B. T. L. (2014). Social Connectedness Predicts Lower Loneliness and Depressive Symptoms over Time in Adolescents. Open Journal of Depression, 03(04), 154-163. https://doi.org/10.4236/ojd.2014.34019

Kent de Grey, R. G., Uchino, B. N., Baucom, B. R., Smith, T. W., Holton, A. E., \& Diener, E. F. (2019). Enemies and friends in high-tech places: The development and validation of the Online Social Experiences Measure. DIGITAL HEALTH, 5, 2055207619878351. https://doi.org/10.1177/2055207619878351

Kessler, R. C., Petukhova, M., Sampson, N. A., Zaslavsky, A. M., \& Wittchen, H. (2012). Twelve-month and lifetime prevalence and lifetime morbid risk of anxiety and mood disorders in the United States. International Journal of Methods in Psychiatric Research, 21(3), 169-184. https://doi.org/10.1002/mpr.1359

Klineberg, E., Clark, C., Bhui, K. S., Haines, M. M., Viner, R. M., Head, J., Woodley-Jones, D., \& Stansfeld, S. A. (2006). Social support, ethnicity and mental health in adolescents. Social Psychiatry \& Psychiatric Epidemiology, 41(9), 755-760. https://doi.org/10.1007/s00127006-0093-8

Kross, E., Verduyn, P., Sheppes, G., Costello, C. K., Jonides, J., \& Ybarra, O. (2020). Social Media and Well-Being: Pitfalls, Progress, and Next Steps. Trends in Cognitive Sciences, $O(0)$. https://doi.org/10.1016/j.tics.2020.10.005 
ADOLESCENT LONELINESS AND ONLINE SOCIAL EXPERIENCES IN COVID-19

Laursen, B., \& Hartl, A. C. (2013). Understanding loneliness during adolescence: Developmental changes that increase the risk of perceived social isolation. Journal of Adolescence, 36(6), 1261-1268. https://doi.org/10.1016/j.adolescence.2013.06.003

Leary, M. R. (1990). Responses to social exclusion: Social anxiety, jealousy, loneliness, depression, and low self-esteem. Journal of Social and Clinical Psychology, 9(2), 221-229. https://doi.org/10.1521/jscp.1990.9.2.221

Lee, S. J. (2009). Online Communication and Adolescent Social Ties: Who benefits more from Internet use?*. Journal of Computer-Mediated Communication, 14(3), 509-531. https://doi.org/10.1111/j.1083-6101.2009.01451.x

Lenhart, A. (2015). Social Media and Teen Friendships. Pew Research Center: Internet, Science \& Tech. https://www.pewresearch.org/internet/2015/08/06/chapter-4-social-media-andfriendships/

Lin, S., Liu, D., Niu, G., \& Longobardi, C. (2020). Active Social Network Sites Use and Loneliness: The Mediating Role of Social Support and Self-Esteem. Current Psychology. https://doi.org/10.1007/s12144-020-00658-8

Loades, M. E., Chatburn, E., Higson-Sweeney, N., Reynolds, S., Shafran, R., Brigden, A., Linney, C., McManus, M. N., Borwick, C., \& Crawley, E. (2020). Rapid Systematic Review: The Impact of Social Isolation and Loneliness on the Mental Health of Children and Adolescents in the Context of COVID-19. Journal of the American Academy of Child and Adolescent Psychiatry, 59(11), 1218-1239.e3. https://doi.org/10.1016/j.jaac.2020.05.009

Lykes, V. A., \& Kemmelmeier, M. (2014). What predicts loneliness? Cultural difference between individualistic and collectivistic societies in Europe. Journal of Cross-Cultural Psychology, 45(3), 468-490. https://doi.org/10.1177/0022022113509881

Maes, M., Qualter, P., Vanhalst, J., Noortgate, W. V. den, \& Goossens, L. (2019). Gender Differences in Loneliness Across the Lifespan: A Meta-Analysis. European Journal of Personality, 33(6), 642-654. https://doi.org/10.1002/per.2220

Magis-Weinberg, L. (2021). Digital citizenship under lockdown: Promoting healthy use of technology for adolescents growing up in Peru during COVID-19. [Special issue: The Clarity of Crisis]. Journal of Children and Media. 
ADOLESCENT LONELINESS AND ONLINE SOCIAL EXPERIENCES IN COVID-19

Magis-Weinberg, L., Berger, E., Gys, C., \& Dahl, R. (2021, February 15). Study Protocol:

Transitions, a school based intervention to promote digital citizenship in low-and middle income secondary school students in Perú. Available. https://doi.org/10.31219/osf.io/gzc82

Magis-Weinberg, L., Suleiman A.B., \& Dahl, R., (2021). Context, development and digital media: implications for very young adolescents in LMICs. Frontiers in Psychology. Special issue on Digital Media and Social Connection in the Lives of Children, Adolescents and Families. Frontiers in Psychology, 12:632713. doi: 10.3389/fpsyg.2021.632713

Mahon, N. E., Yarcheski, A., \& Yarcheski, T. J. (2001). Mental Health Variables and Positive Health Practices in Early Adolescents. Psychological Reports, 88, 1023-1030. https://doi.org/10.2466/pr0.2001.88.3c.1023

Meeus, W. (1989). Parental and peer support in adolescence. In The social world of adolescents: International perspectives (pp. 167-183). Walter De Gruyter.

Morahan-Martin, J., \& Schumacher, P. (2003). Loneliness and social uses of the Internet. Computers in Human Behavior, 19(6), 659-671. https://doi.org/10.1016/S0747$\underline{5632(03) 00040-2}$

Na, J., Kosinski, M., \& Stillwell, D. J. (2015). When a New Tool Is Introduced in Different Cultural Contexts: Individualism-Collectivism and Social Network on Facebook. Journal of Cross-Cultural Psychology, 46(3), 355-370. https://doi.org/10.1177/0022022114563932

Nakagawa, S., Johnson, P. C. D., \& Schielzeth, H. (2017). The coefficient of determination R2 and intra-class correlation coefficient from generalized linear mixed-effects models revisited and expanded. Journal of the Royal Society, Interface, 14(134). https://doi.org/10.1098/rsif.2017.0213

Nelson, E. E., Jarcho, J. M., \& Guyer, A. E. (2015). Social re-orientation and brain development: An expanded and updated view. Developmental Cognitive Neuroscience, 17, 118-127. https://doi.org/10.1016/j.den.2015.12.008

Neto, F., \& Barros, J. (2000). Predictors of loneliness among adolescents from Portuguese immigrant families in Switzerland. Social Behavior and Personality, 28(2), 193-206. https://doi.org/10.2224/sbp.2000.28.2.193

Newman, B. M., Newman, P. R., Griffen, S., O’Connor, K., \& Spas, J. (2007). The relationship of social support to depressive symptoms during the transition to high school. Adolescence, 42(167), 441-459. 
ADOLESCENT LONELINESS AND ONLINE SOCIAL EXPERIENCES IN COVID-19

Odgers, C. L., \& Jensen, M. R. (2020). Annual Research Review: Adolescent mental health in the digital age: facts, fears, and future directions. Journal of Child Psychology and Psychiatry, 61(3), 336-348. https://doi.org/10.1111/jcpp.13190

OECD. (2019). Society at a Glance 2019. https://www.oecdilibrary.org/content/publication/soc glance-2019-en

Orben, A. (2020). Teenagers, screens and social media: A narrative review of reviews and key studies. Social Psychiatry and Psychiatric Epidemiology, 55(4), 407-414. https://doi.org/10.1007/s00127-019-01825-4

Orben, A., Tomova, L., \& Blakemore, S.-J. (2020). The effects of social deprivation on adolescent development and mental health. The Lancet Child \& Adolescent Health, 4(8), 634-640. https://doi.org/10.1016/S2352-4642(20)30186-3

Perlman, D., \& Peplau, L. A. (1981). Toward a Social Psychology of Loneliness. Personal Relationships, 3, 31-56.

Primack, B. A., Karim, S. A., Shensa, A., Bowman, N., Knight, J., \& Sidani, J. E. (2019). Positive and Negative Experiences on Social Media and Perceived Social Isolation. American Journal of Health Promotion, 33(6), 859868. https://doi.org/10.1177/0890117118824196

Primack, B., Shensa, A., Escobar-Viera, C., Barrett, E., Sidani, J., Colditz, J., \& James, A. (2017). Use of multiple social media platforms and symptoms of depression and anxiety: A nationally-representative study among U.S. young adults. Computers in Human Behavior, 69, 1-9. https://doi.org/10.1016/j.chb.2016.11.013

Przybylski, A. K., \& Weinstein, N. (2017). A Large-Scale Test of the Goldilocks Hypothesis: Quantifying the Relations Between Digital-Screen Use and the Mental Well-Being of Adolescents. Psychological Science, 28(2), 204-215. https://doi.org/10.1177/0956797616678438

Purwono, U., \& French, D. (2016). Depression and its relation to loneliness and religiosity in Indonesian Muslim adolescents. Mental Health, Religion \& Culture, 19, 1-11. https://doi.org/10.1080/13674676.2016.1165190

Qi, M., Zhou, S.-J., Guo, Z.-C., Zhang, L.-G., Min, H.-J., Li, X.-M., \& Chen, J.-X. (2020). The Effect of Social Support on Mental Health in Chinese Adolescents During the Outbreak of 


\section{ADOLESCENT LONELINESS AND ONLINE SOCIAL EXPERIENCES IN COVID-19}

COVID-19. Journal of Adolescent Health, 67(4), 514-518.

https://doi.org/10.1016/j.jadohealth.2020.07.001

Qualter, P., Rotenberg, K., Barrett, L., Henzi, P., Hennessey, A., Stylianou, M., \& Nowland, R. (2012). Investigating Hypervigilance for Social Threat of Lonely Children. Journal of Abnormal Child Psychology. https://doi.org/10.1007/s10802-012-9676-X

Qualter, P., Vanhalst, J., Harris, R., Van Roekel, E., Lodder, G., Bangee, M., Maes, M., \& Verhagen, M. (2015). Loneliness Across the Life Span. Perspectives on Psychological Science, 10(2), 250-264. https://doi.org/10.1177/1745691615568999

Rideout, V., \& Fox, S. (2018). Digital Health Practices, Social Media Use, and Mental WellBeing Among Teens and Young Adults in the U.S. Hope Lab and Well Being Trust, 95.

Rokach, A., \& Neto, F. (2000). Causes of Loneliness in Adolescence: A Cross-Cultural Study. International Journal of Adolescence and Youth, 8(1), 65-80.

https://doi.org/10.1080/02673843.2000.9747842

Rose, C. A., \& Tynes, B. M. (2015). Longitudinal Associations Between Cybervictimization and Mental Health Among U.S. Adolescents. The Journal of Adolescent Health: Official Publication of the Society for Adolescent Medicine, 57(3), 305-312.

https://doi.org/10.1016/j.jadohealth.2015.05.002

Russell, D. W. (1996). UCLA Loneliness Scale (Version 3): Reliability, Validity, and Factor Structure. Journal of Personality Assessment, 66(1), 20-40.

https://doi.org/10.1207/s15327752jpa6601 2

Shensa, A., Sidani, J. E., Dew, M. A., Escobar-Viera, C. G., \& Primack, B. A. (2018). Social Media Use and Depression and Anxiety Symptoms: A Cluster Analysis. American Journal of Health Behavior, 42(2), 116-128. https://doi.org/10.5993/AJHB.42.2.11

Shovestul, B., Han, J., Germine, L., \& Dodell-Feder, D. (2020). Risk factors for loneliness: The high relative importance of age versus other factors. PLOS ONE, 15(2), e0229087. https://doi.org/10.1371/journal.pone.0229087

Smirni, P., Lavanco, G., \& Smirni, D. (2020). Anxiety in Older Adolescents at the Time of COVID-19. Journal of Clinical Medicine, 9(10). https://doi.org/10.3390/jcm9103064

Song, H., Zmyslinski-Seelig, A., Kim, J., Drent, A., Victor, A., Omori, K., \& Allen, M. (2014). Does Facebook make you lonely?: A meta analysis. Computers in Human Behavior, 36, 446-452. https://doi.org/10.1016/j.chb.2014.04.011 
ADOLESCENT LONELINESS AND ONLINE SOCIAL EXPERIENCES IN COVID-19

Stanley, D. J., \& Spence, J. R. (2018). Reproducible Tables in Psychology Using the apaTables Package. Advances in Methods and Practices in Psychological Science, 1(3), 415-431. https://doi.org/10.1177/2515245918773743

Stickley, A., Koyanagi, A., Koposov, R., Schwab-Stone, M., \& Ruchkin, V. (2014). Loneliness and health risk behaviours among Russian and U.S. adolescents: A cross-sectional study. BMC Public Health, 14, 366. https://doi.org/10.1186/1471-2458-14-366

Subirana, I., Sanz, H., \& Vila, J. (2014). Building Bivariate Tables: The compareGroups Package for R. Journal of Statistical Software, 57. https://doi.org/10.18637/jss.v057.i12

Teppers, E., Luyckx, K., A. Klimstra, T., \& Goossens, L. (2014). Loneliness and Facebook motives in adolescence: A longitudinal inquiry into directionality of effect. Journal of Adolescence, 37(5), 691-699. https://doi.org/10.1016/j.adolescence.2013.11.003

Uhls, Y. T., Ellison, N. B., \& Subrahmanyam, K. (2017). Benefits and Costs of Social Media in Adolescence. Pediatrics, 140(Supplement 2), S67-S70. https://doi.org/10.1542/peds.2016$\underline{1758 \mathrm{E}}$

Valkenburg, P. M., \& Peter, J. (2008). Adolescents' Identity Experiments on the Internet: Consequences for Social Competence and Self-Concept Unity. Communication Research, 35(2), 208-231. https://doi.org/10.1177/0093650207313164

van den Eijnden, R. J. J. M., Meerkerk, G.-J., Vermulst, A. A., Spijkerman, R., \& Engels, R. C. M. E. (2008). Online communication, compulsive internet use, and psychosocial well-being among adolescents: A longitudinal study. Developmental Psychology, 44(3), 655-665. https://doi.org/10.1037/0012-1649.44.3.655

van Staden, W. (C. W.), \& Coetzee, K. (2010). Conceptual relations between loneliness and culture. Current Opinion in Psychiatry, 23(6), 524-529. https://doi.org/10.1097/YCO.0b013e32833f2ff9

Vannucci, A., \& McCauley Ohannessian, C. (2019). Social Media Use Subgroups Differentially Predict Psychosocial Well-Being During Early Adolescence. Journal of Youth and Adolescence, 48(8), 1469-1493. https://doi.org/10.1007/s10964-019-01060-9

von Soest, T., Luhmann, M., \& Gerstorf, D. (2020). The development of loneliness through adolescence and young adulthood: Its nature, correlates, and midlife outcomes. Developmental Psychology, 56(10), 1919-1934. https://doi.org/10.1037/dev0001102 


\section{ADOLESCENT LONELINESS AND ONLINE SOCIAL EXPERIENCES IN COVID-19}

Wang, J., Lloyd-Evans, B., Giacco, D., Forsyth, R., Nebo, C., Mann, F., \& Johnson, S. (2017). Social isolation in mental health: A conceptual and methodological review. Social Psychiatry and Psychiatric Epidemiology, 52(12), 1451-1461. https://doi.org/10.1007/s00127-017-1446-1

Williams, K. D., \& Zadro, L. (2005). Ostracism: The indiscriminate early detection system. In The social outcast: Ostracism, social exclusion, rejection, and bullying (pp. 19-34). Psychology Press.

Wood, D., Larson, R. W., \& Brown, J. R. (2009). How Adolescents Come to See Themselves as More Responsible Through Participation in Youth Programs. Child Development, 80(1), 295-309. https://doi.org/10.1111/j.1467-8624.2008.01260.x

Yau, J., \& Reich, S. (2017). Are the Qualities of Adolescents' Offline Friendships Present in Digital Interactions? Adolescent Research Review, 3. https://doi.org/10.1007/s40894-0170059-y

Yum, Y.-O. (2003). The relationships among loneliness, self/partner constructive maintenance behavior, and relational satisfaction in two cultures. Communication Studies, 54(4), 451467. https://doi.org/10.1080/10510970309363303

Zhou, S.-J., Zhang, L.-G., Wang, L.-L., Guo, Z.-C., Wang, J.-Q., Chen, J.-C., Liu, M., Chen, X., \& Chen, J.-X. (2020). Prevalence and socio-demographic correlates of psychological health problems in Chinese adolescents during the outbreak of COVID-19. European Child \& Adolescent Psychiatry, 29(6), 749-758. https://doi.org/10.1007/s00787-020-01541-4

Zimet, G. (2016). Multidimensional Scale of Perceived Social Support (MSPSS)—Scale Items and Scoring Information. 